\title{
Atomistic spin model simulation of magnetic reversal modes near the Curie point
}

\author{
J. Barker, ${ }^{1, a)}$ R. F. L. Evans, ${ }^{1}$ R. W. Chantrell, ${ }^{1}$ D. Hinzke, ${ }^{2}$ and U. Nowak ${ }^{2}$ \\ ${ }^{1}$ Department of Physics, The University of York, York YO10 5DD, United Kingdom \\ ${ }^{2}$ Fachbereich Physik, Universität Konstanz, Universitätsstrasse 10, D-78464 Konstanz, Germany
}

\begin{abstract}
The so-called linear reversal mode is demonstrated in spin model simulations of the high anisotropy material $\mathrm{L} 1_{0} \mathrm{FePt}$. Reversal of the magnetization is found to readily occur in the linear regime despite an energy barrier $\left(K V / k_{\mathrm{B}} T\right)$ that would conventionally ensure stability on this timescale. The timescale for the reversal is also established with a comparison to the Landau-Lifshitz-Bloch equation showing good agreement.
\end{abstract}

In order for the current increase in magnetic storage density to continue, one must overcome the so-called magnetic recording trilemma; namely, that smaller grains are required for higher data densities and to ensure their thermal stability, materials with a high anisotropy are required. The higher coercive field that this produces also becomes a limiting factor as the maximum field produced by the recording head is constrained by the saturation magnetization of the pole. One proposed solution to the trilemma is the use of heat assisted magnetic recording (HAMR), which utilizes the temperature dependence of the anisotropy to enable writing of materials with a high coercivity. For the highest anisotropy media, this will require heating to the Curie temperature $\left(T_{\mathrm{C}}\right)$ of the material. Close to $T_{\mathrm{C}}$, longitudinal fluctuations in the magnetization can have a significant impact on the expected energy barriers and therefore the relaxation time of the magnetization. These effects become especially important when attempting to minimize the time to reverse the magnetization state of the media that will be important at higher storage densities.

Recently, the existence of a so-called linear reversal mode was predicted ${ }^{1}$ from the Landau-Lifshitz-Bloch (LLB) equation. ${ }^{2}$ During linear reversal, the magnetization does not coherently rotate, but instead linearly reduces along the easy axis, reappearing in the opposite sense in the same manner. This reversal mode is found in materials with a very high anisotropy and only occurs close to $T_{\mathrm{C}}$ (although at temperatures less than $T_{\mathrm{C}}$ ), where this reduction of magnetization becomes more energetically favorable than coherent rotation. Analytic work by Kazantseva et al. ${ }^{1}$ with the LLB equation has suggested that linear reversal occurs on a much faster timescale than coherent rotation.

The linear reversal mechanism seems to be a contributory factor in the optomagnetic reversal phenomenon observed by Stanciu et al. ${ }^{3}$ These experiments circularly used a polarized laser light to demonstrate magnetization reversal using $100 \mathrm{fs}$ pulses in the absence of an externally applied field. This is a timescale that is much shorter than expected for magnetization reversal by precession, but one accessible to the linear reversal mechanism according to the model of Kazantseva et al. ${ }^{1}$ Using a model based on the LLB equation, Vahaplar et al. ${ }^{4}$ showed that reversal on a subpicosecond timescale is possible via the linear reversal mechanism

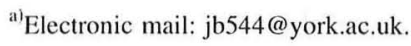

and experiments supported the predicted criticality of the onset of the linear reversal mechanism, which occurs at a temperature determined by the ratio of the longitudinal and transverse susceptibilities. ${ }^{1}$ Thus linear reversal seems to be central to the optomagnetic reversal mechanism.

Consequently, linear reversal is an important mechanism, justifying detailed investigation of its physical basis. In this letter, we use atomistic scale dynamic simulations to demonstrate the existence of linear and elliptical reversal modes. We also show that reversal readily occurs where conventionally, a Stoner-Wohlfarth type barrier $\left(K V / k_{\mathrm{B}} T\right)$ would ensure thermal stability on a long timescale. Finally, we make a direct comparison between the reversal times in the atomistic spin simulation and the values calculated using the LLB equation.

The model Hamiltonian uses the Heisenberg form of exchange for moments well localized to atomic sites. It is important in this work to have such microscopic detail so that temperatures close to the Curie point and through the phase transition can be reproduced in the model. In this paper we model the high anisotropy material $\mathrm{L1}_{0}$ ordered FePt. This material is known to have a very large uniaxial magnetic anisotropy of $K \approx 10^{8} \mathrm{erg} / \mathrm{cc}$, making it a good candidate for next generation hard drive devices. ${ }^{5}$ The model is parameterized with $a b$ initio data for the exchange interaction and anisotropy as found by Mryasov et al. ${ }^{6}$ The large anisotropy in $\mathrm{L} 1_{0} \mathrm{FePt}$ arises due to the two-ion exchange that exists between the alternating layers of Fe and Pt. Mryasov et al. showed that the moment induced in the Pt ions has a direction and magnitude that is linearly dependent on the exchange field from the surrounding $\mathrm{Fe}$. This allows the $\mathrm{Pt}$ spins to be combined onto the Fe lattice sites. The result is a Hamiltonian that only contains Fe spins, $\boldsymbol{S}_{i}$, but has a long range exchange that is mediated by the $\mathrm{Pt}$ sites. ${ }^{6}$ Equation (1) gives the Hamiltonian, where $\tilde{J}_{i j}$ is the effective exchange, $d_{i}^{(0)}$ is the single ion anisotropy energy and $d_{i j}^{(2)}$ is the two-ion anisotropy energy, $\boldsymbol{H}$ is the applied field, and $\widetilde{\mu}=\mu \mathrm{Fe}+\mu \mathrm{Pt}$

$$
\begin{aligned}
\mathcal{H}= & -\tilde{J}_{i j} \sum_{i \neq j} S_{i} \cdot S_{j}-d_{i}^{(0)} \sum_{i}\left(S_{i}^{z}\right)^{2}-d_{i j}^{(2)} \sum_{i \neq j} S_{i}^{z} S_{j}^{z} \\
& -\tilde{\mu} \mathbf{H} \cdot \sum_{i} S_{i} .
\end{aligned}
$$

Due to the dependence of the anisotropy on the ordering of 
the FePt, perfect $\mathrm{L1}_{0}$ phase is assumed by the model, as is a 1:1 stoichiometry.

The $a b$ initio characterization in this model produces a ferromagnetic state with a Curie temperature of $T_{\mathrm{C}}$ $\approx 700 \mathrm{~K}$ for a bulk (periodic) system that is just slightly lower than the experimentally observed value of $T_{\mathrm{C}}$ $=750 \mathrm{~K} .{ }^{7}$ For granular (open) systems, the atomic sites are characterized using the same long ranged exchange and two-ion anisotropic energies as for the bulk, but the total exchange on many sites is reduced due to the absence of interacting neighboring spin sites at the surface. This approximation is used in the absence of detailed experimental or $a b$ initio characterization of FePt surfaces. It is noted that because the large anisotropy depends on the long range twoion anisotropy, this will also be reduced at the surface.

The dynamics of each atomistic moment is described by the Landau-Lifshitz-Gilbert (LLG) equation,

$$
\frac{\partial \boldsymbol{S}_{i}}{\partial t}=-\frac{\gamma}{\left(1+\lambda^{2}\right) \mu_{s}} \boldsymbol{S}_{i} \times\left\{\boldsymbol{H}_{i}(t)+\lambda\left[\boldsymbol{S}_{i} \times \boldsymbol{H}_{i}(t)\right]\right\} .
$$

The effective field on each spin is given by $\boldsymbol{H}_{i}=-\lambda \mathcal{H} / \lambda \mathbf{S}_{i}$ $+\zeta_{i}$, where $\zeta_{i}$ are three independent stochastic processes that satisfy the conditions

$$
\begin{aligned}
& \left\langle\zeta_{i}\right\rangle=0 ; \\
& \left\langle\zeta_{i}(t) \zeta_{j}\left(t^{\prime}\right)\right\rangle=\delta_{i j} \delta\left(t-t^{\prime}\right) 2 \lambda k_{\mathrm{B}} T \mu_{s} / \gamma,
\end{aligned}
$$

where $\alpha$ is the Gilbert damping parameter; $k_{\mathrm{B}}$ is the Boltzmann constant; and $T$ is the temperature of the thermal heat bath. In ultrafast laser experiments to which this work is comparable, it would be the conduction electron heat bath that this represents. In such experiments, the phonon and electron heat baths are in a highly nonequlibrium state, thus the electron heat bath temperature can be considerably higher than that of the phonons. ${ }^{8,9} \mu_{s}$ is the atomic moment that is $3.23 \mu_{\mathrm{B}}$ for the localized $\mathrm{Fe}+\mathrm{Pt}$ combined moments; $\gamma$ is the gyromagnetic ratio given the value 1.76 $\times 10^{11} \mathrm{rad} \mathrm{s}^{-1} \mathrm{~T}^{-1}$. The LLG equation was integrated using the Heun method with a time step of $0.5 \mathrm{fs}$. For the fast processes being investigated in this paper and the high temporal resolution used, the validity of white noise is questionable. ${ }^{10}$ Recent attempts have been made to include colored noise into the Langevin equation of motion; ${ }^{11}$ however the approach of Atxitia et al. requires at least two unknown parameters, the heat bath correlation time and the bath coupling strength. Therefore we will use white noise thermal processes.

A reversal path for the system was calculated by allowing the system to evolve at thermal equilibrium for a long period of time in zero field. Comparing the mean values of the longitudinal magnetization, $m_{z}$ and transverse magnetization, $m_{t}$, as the magnetization moves through the configuration space, the mean reversal path was obtained. This calculation was performed for both "up" and "down" initial configurations so that a range of motion can be established for systems that do not undergo thermal reversal within the timescale of the simulations.

The results in Fig. 1 are from an ensemble of periodic systems with a total combined integration time of $2 \mathrm{~ns}$. It can be seen that as the system approaches $T_{\mathrm{C}}$, the ellipticity of the mean reversal path increases. Very close to $T_{\mathrm{C}}$, the behavior changes to a linear mode where $m_{t}$ remains very small

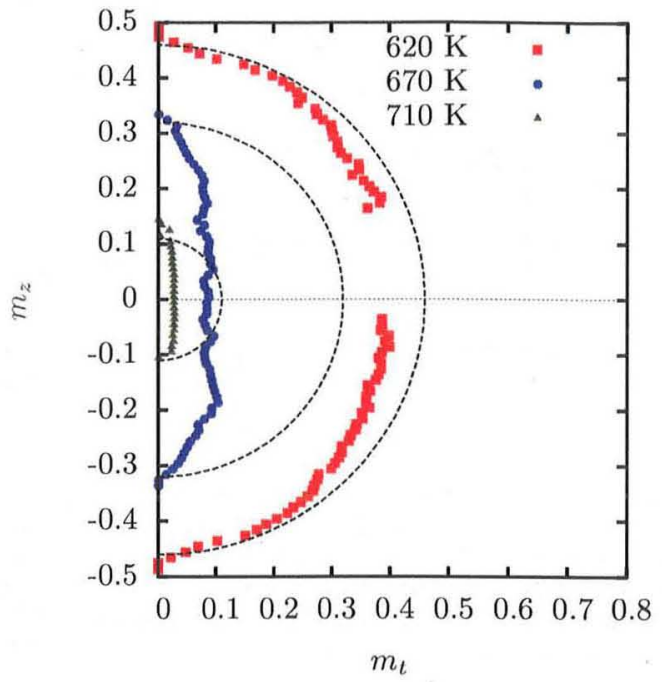

FIG. 1. (Color online) Mean reversal path of a periodic system ( $T_{\mathrm{C}}$ $\approx 700 \mathrm{~K}$ ) showing the equilibrium magnetization vector for given temperatures. Dashed lines are guides representing a circular reversal path for each temperature.

for all values of $m_{z}$. The small remanent $m_{t}$ is a finite size effect. A calculation of the Stoner-Wohlfarth type barrier $\Delta E=K V / k_{\mathrm{B}} T$ gives $\Delta E \approx 100$ for $620 \mathrm{~K}$ and $\Delta E \approx 30$ for $670 \mathrm{~K}$. For both of these values, reversal would be very unlikely within the $2 \mathrm{~ns}$ of total simulation time, yet within a temperature change of $50 \mathrm{~K}$ the system goes from being thermally stable to superparamagnetic, suggesting a dramatic reduction of the energy barrier associated with the onset of the linear reversal mode.

The reversal time is also significant, as it governs the fundamental speed of magnetic phenomena. We have therefore calculated the magnetization reversal time $t_{0}^{1}$ for comparison with the analytic solution of the LLB equation by Kazantseva et al. ${ }^{1}$ for reversal times. The time $t_{0}^{1}$ is defined as the time taken to for the system to change from a state of $m_{z}=1$ to $m_{z}=0$ with a reversing field applied along the easy axis. The results are the average of many simulations to establish a mean reversal time. We now compare the atomistic results with analytic LLB calculations. We note that in reference $^{1}$ the LLB equation has been parameterized for a system size of $6 \mathrm{~nm}$. To allow a direct comparison with the analytical results we have carried out atomistic calculations for a system size of $6 \mathrm{~nm}$ to ensure that the susceptibilities and equilibrium magnetization had the same temperature variation as those used in the LLB model. Due to finite size effects, which are especially pronounced in FePt due to the long range nature of the exchange, this system has a smaller $T_{\mathrm{C}}$ of $600 \mathrm{~K} .^{12}$

Figure 2 shows a very good agreement between the atomistic simulation results and the analytic solution of the LLB equation. ${ }^{1}$ Importantly, the reversal time changes by an order of magnitude across $T_{\mathrm{C}}$ in the linear reversal regime. This effect is significant for HAMR, as it shows that reversal is possible in the magnetically hardest materials, but heating close to $T_{\mathrm{C}}$ will be necessary.

To illustrate this effect more explicitly, we have simulated the reversal probability of a $6 \mathrm{~nm}$ grain under a $10 \mathrm{ps}$ heat pulse and $1 \mathrm{~T}$ field, as shown in Fig. 3. The reversal probability is zero at low temperatures, consistent with the 


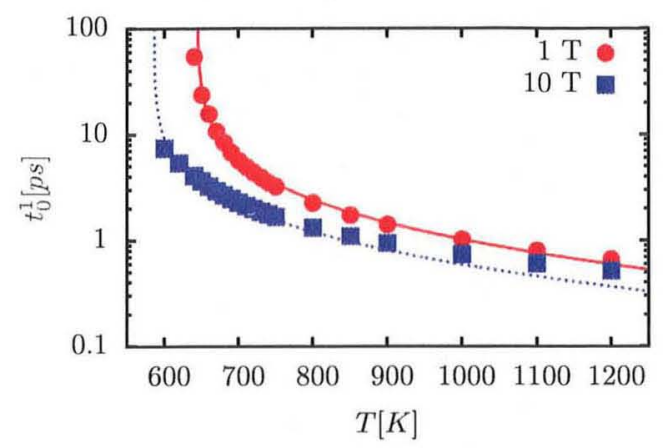

FIG. 2. (Color online) A comparison of the characteristic reversal time $t_{0}^{1}$ as a function of temperature, through $T_{\mathrm{C}}$ in a $6 \mathrm{~nm}$ cube of $\mathrm{FePt}\left(T_{\mathrm{C}}=660 \mathrm{~K}\right.$ for this small finite size system). Reversing fields (an applied field along the $z$-axis opposing the magnetization) of 1 and $10 \mathrm{~T}$ are compared. Atomistic spin simulations are represented by points and the solid lines are the analytic solution of the LLB equation (see Ref. 1).

large energy barriers noted earlier. At a critical temperature of $\sim 640 \mathrm{~K}$, the reversal probability increases rapidly. Note that this temperature is consistent with the estimate of $\sim 642 \mathrm{~K}$ for the critical temperature $T$ for the onset of linear reversal. $T^{*}$ is determined by the condition $\tilde{\chi}_{\|} / \widetilde{X}_{1}=1 / 2$, where $\tilde{\chi}_{\|}, \tilde{\chi}_{\perp}$ are the longitudinal and transverse susceptibilities respectively. We note that the maximum reversal probability reached is less than unity. This reflects the thermal equilibrium probability, which is determined by $\tanh (\mu H / k T)$ where $\mu$ is the total spin moment of the nanoparticle. The sharp transition at the critical temperature favorably compares with Fig. 2 and the results of Kazantseva et al. Importantly, the criticality of the reversal mechanism is

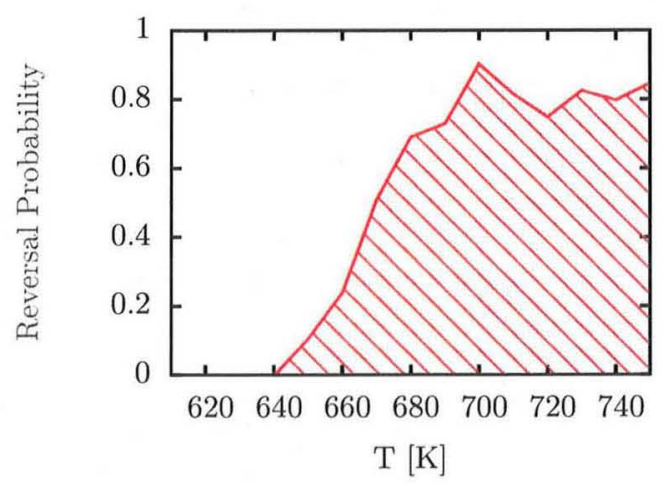

FIG. 3. (Color online) The reversal probability of a $6 \mathrm{~nm}$ FePt grain in a 1 $\mathrm{T}$ reversing field after the application of a $10 \mathrm{ps}$ square heat pulse. The shaded area represents reversal $\left(m_{z}<0\right)$. demonstrated in agreement with the experimental data of Vahaplar et al. ${ }^{4}$

In summary, we have demonstrated the so-called linear reversal mode within atomistic spin model simulations. This has shown that in the linear regime, reversal occurs within a timescale much shorter than the expected relaxation time for the conventional Stoner-Wohlfarth barrier $\left(K V / k_{\mathrm{B}} T\right)$. The onset of this linear regime also appears to be very critical with thermal stability and reversal being separated by a relatively small change in temperature.

The atomistic spin simulations performed here support the analytic solution of the LLB equation by Kazantseva et al. with respect to reversal times. Again these results confirm the apparent criticality of the onset of the linear reversal mode. Very close to $T_{\mathrm{C}}$ the reversal time of the system changes by at least an order of magnitude. These results also demonstrate that for temperatures and fields achievable in the nonequilibrium regime of ultrafast laser experiments, reversal is possible on a subpicosecond timescale, which is consistent with the optomagnetic reversal experiments of Stanciu et al. ${ }^{3}$

J.B. is grateful to the EPSRC Contract No. EP/ P505178/1 for provision of a PhD studentship. D.H. acknowledges support by the Deutsche Forschungsgemeinschaft through Grant No. SFB 767. Financial support of the EU FP7 program [Grant No. NMP3-SL-2008-214469 (UltraMagnetron) and Grant No. 214810 (FANTOMAS)] is also gratefully acknowledged.

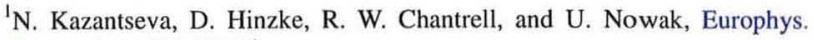
Lett. 86, 27006 (2009).

${ }^{2}$ D. A. Garanin, Phys. Rev. B 55, 3050 (1997).

${ }^{3}$ C. D. Stanciu, F. Hansteen, A. V. Kimel, A. Kirilyuk, A. Tsukamoto, A. Itoh, and Th. Rasing, Phys. Rev. Lett. 99, 047601 (2007).

${ }^{4}$ K. Vahaplar, A. M. Kalashnikova, A. V. Kimel, D. Hinzke, U. Nowak, R. Chantrell, A. Tsukamoto, A. Itoh, A. Kirilyuk, and Th. Rasing, Phys. Rev. Lett. 103, 117201 (2009).

${ }^{5}$ D. Weller, A. Moser, L. Folks, M. E. Best, W. Lee, M. F. Toney, M. Schwickert, J.-U. Thiele, and M. F. Doerner, IEEE Trans. Magn. 36, 10 (2000).

${ }^{6}$ O. N. Mryasov, U. Nowak, K. Y. Guslienko, and R. W. Chantrell, Europhys. Lett. 69, 805 (2005).

${ }^{7}$ S. Okamoto, N. Kikuchi, O. Kitakami, T. Miyazaki, and Y. Shimada, Phys. Rev. B 66, 024413 (2002).

${ }^{8}$ P. B. Allen, Phys. Rev. Lett. 59, 1460 (1987).

${ }^{9}$ N. Kazantseva, U. Nowak, R. W. Chantrell, J. Hohlfeld, and A. Rebei, Europhys. Lett. 81, 27004 (2008).

${ }^{10}$ W. F. Brown, Phys. Rev. 130, 1677 (1963).

${ }^{11}$ U. Atxitia, O. Chubykalo-Fesenko, R. W. Chantrell, U. Nowak, and A. Rebei, Phys. Rev. Lett. 102, 057203 (2009).

${ }^{12}$ U. Nowak, O. N. Mryasov, R. Wieser, K. Guslienko, and R. W. Chantrell, Phys. Rev. B 72, 172410 (2005). 